\title{
INTERFEROMETRIC IMAGE RECONSTRUCTION AS A NONLINEAR BAYESIAN ESTIMATION PROBLEM
}

\author{
José M. N. Leitão and Mário A. T. Figueiredo \\ Instituto de Telecomunicações, and \\ Departamento de Engenharia Electrotécnica e de Computadores. \\ Instituto Superior Técnico, 1096 Lisboa Codex, PORTUGAL
}

\begin{abstract}
This paper formulates interferometric image reconstruction as a $2 \mathrm{D}$ absolute phase estimation problem. The original phase image is modeled as a sample of a Gauss Markov random field; the observations are the noisy in-phase (cosine) and quadrature (sine) images. The proposed solution combines features of the iterated conditional modes algorithm with nonlinear stochastic absolute phase estimation concepts. Examples of important applications are: interferometric synthetic aperture radar, optical interferometry, magnetic resonance imaging, and diffraction tomography.
\end{abstract}

\section{INTRODUCTION}

In interferometric imaging techniques, information concerning the observed objects is inferred from absolute (not simply modulo $2 \pi$ ) phase measurements:

- In interferometric synthetic aperture radar (InSAR), phase measurements are used to produce topographic maps; specifically, phase differences are proportional to the elevation of the observed terrain (see, e.g., [1] and [2]).

- Similarly, in optical interferometric imaging, phase differences are used to infer the position of each point of a surface under inspection (see, e.g., [3] and [4]).

- In magnetic resonance imaging (MRI), absolute phase measurements are necessary, e.g., to increase the dynamic range of phase contrast velocity images [5], [6].

- In diffraction tomography, to obtain the complex logarithm of a normalized field, the determination of its absolute phase is needed [7].

Conventional techniques use a two step procedure:

1) determination of modulo $2 \pi$ phase values (the wrapped phases), i.e. the so-called interferogram;

2) phase unwrapping based on some heuristic or ad hoc surface (phase) continuity criterion.

To deal with interferometric image reconstruction in a systematic way, a Bayesian estimation approach is adopted in this paper. Accordingly, a probabilistic observation model and structured prior knowledge concerning the original image are needed.

\section{PROBLEM FORMULATION}

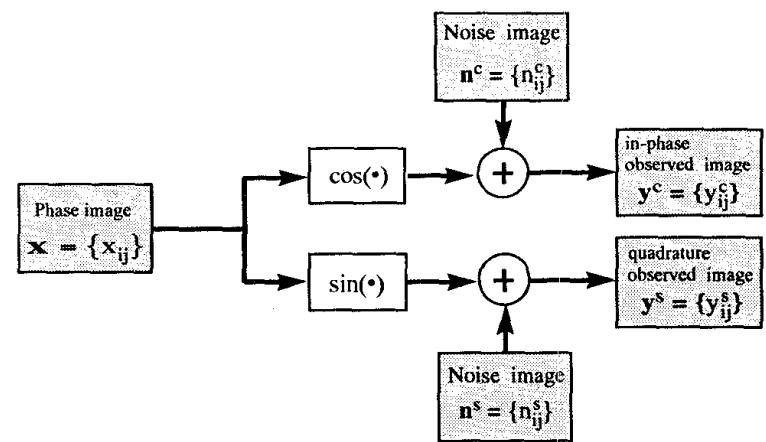

Figure 1: Base-band observation model of interferometric imaging techniques.

\subsection{Observation model}

The (base-band) observation model depicted in Fig. 1 captures the essential features of the data acquisition mechanisms used in the class of problems we are considering. The observations $\mathbf{y}^{c}=\left\{y_{i j}^{c}\right\}$ and $\mathbf{y}^{s}=\left\{y_{i j}^{s}\right\}$ are the inphase (cosine) and quadrature (sine) images, associated to the phase field $\mathbf{x}=\left\{x_{i j}\right\}$, with additive white Ganssian independent noises $\mathbf{n}^{c}=\left\{x_{i j}^{c}\right\}$ and $\mathbf{n}^{s}=\left\{x_{i j}^{s}\right\}$, respectively; for simplicity, homogeneous noises of variance $\sigma^{2}$ are assumed. The (pixel-wise) observation model is then

$$
\left[\begin{array}{l}
y_{i j}^{c} \\
y_{i j}^{s}
\end{array}\right]=\left[\begin{array}{c}
\cos \left(x_{i j}\right) \\
\sin \left(x_{i j}\right)
\end{array}\right]+\left[\begin{array}{c}
n_{i j}^{c} \\
n_{i j}^{s}
\end{array}\right] \text {. }
$$

\subsection{Original image model}

We take the original image/surface as a sample of a causal Gauss-Markov random field (GMRF) [8], [9], specifically, the one generated according to the following $2 \mathrm{D}$ autoregressive (AR) model

$$
x_{i j}=\frac{1}{2} x_{i j-1}+\frac{1}{2} x_{i-1 j}+u_{i j},
$$

where the $u_{i j}$ 's are i.i.d. Gaussian variables of zero mean and variance $\mu^{2}$. Although simple, model (2) expresses surface continuity in a formal way, and can easily be extended in several directions. 
Fig. 2 shows the original phase field generated according to model (2), with $\mu=0.7$, plus a deterministic Gaussianshaped surface in the central region. Fig. 3 presents the noisy in-phase (cosine) and quadrature (sine) observations from the surface of Fig. 2, according to (1) with $\sigma=0.3$.

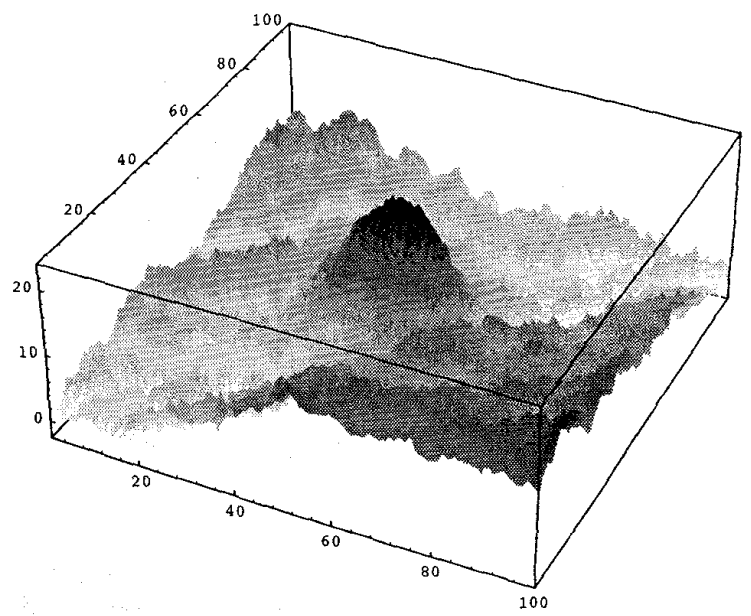

Figure 2: Original phase surface/field.

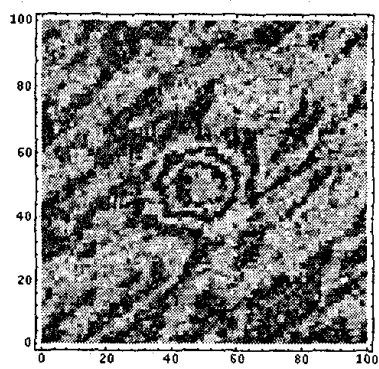

Figure 3: Noisy in-phase (right) observed images.

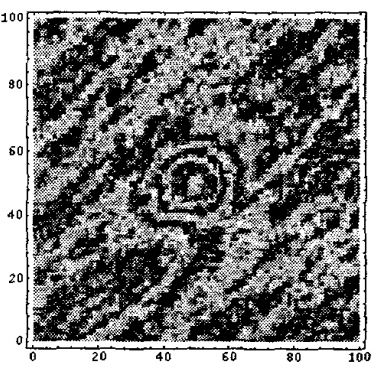

(left) and quadrature

\subsection{Estimation criterion}

The problem consists in estimating the original image based on the models and assumptions above stated. We adopt the maximum a posteriori (MAP) estimation criterion, i.e.,

$\widehat{\mathbf{x}}_{\text {MAP }}=\arg \max _{\mathbf{X}}\left\{p\left(\mathbf{x} \mid \mathbf{y}^{c}, \mathbf{y}^{s}\right)\right\}=\arg \max _{\mathbf{X}}\left\{p\left(\mathbf{y}^{c}, \mathbf{y}^{s} \mid \mathbf{x}\right) p(\mathbf{x})\right\}$,

where $p(\mathbf{x})$ is the joint probability density function of the causal GMRF and

$$
p\left(\mathbf{y}^{c}, \mathbf{y}^{s} \mid \mathbf{x}\right)=\prod_{i j} p\left(y_{i j}^{c}, y_{i j}^{s} \mid x_{i j}\right)
$$

where, from (1),

$$
p\left(y_{i j}^{c}, y_{i j}^{s} \mid x_{i j}\right) \propto \exp \left\{\lambda_{i j} \cos \left(x_{i j}-\eta_{i j}\right)\right\}
$$

with

$$
\begin{aligned}
\lambda_{i j} & =\frac{1}{\sigma^{2}} \sqrt{\left(y_{i j}^{c}\right)^{2}+\left(y_{i j}^{c}\right)^{2}} \\
\eta_{i j} & =\arctan \left(\frac{y_{i j}^{\mathrm{c}}}{y_{i j}^{s}}\right) .
\end{aligned}
$$

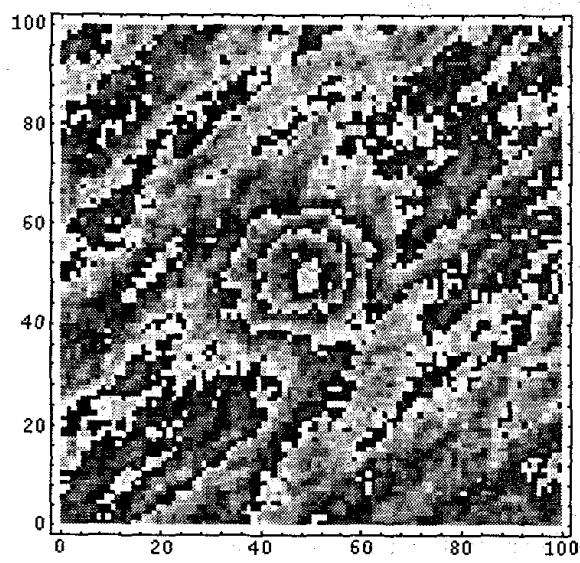

Figure 4: Interferogram computed from the images of Fig.3, according to (7).

Fig. 4 shows the interferogram whose pixels are computed according to (7).

\section{PROPOSED SOLUTION}

\subsection{The approach}

To reconstruct $\mathbf{x}$ from the observations $\mathbf{y}^{c}$ and $\mathbf{y}^{s}$, we developed a sub-optimal approach which inherits features from the iterated conditional modes (ICM) scheme [10] and from the nonlinear stochastic phase estimation methodology proposed in [11] and [12].

As in ICM, each pixel estimate is the maximizer of its conditional probability density function, given its neighbor estimates and the associated observation; the model expressed in (2) implies a causal two-nearest-neighbors structure yielding

$$
\widehat{x}_{i j}=\arg \max _{x_{i j}} p\left(x_{i j} \mid \widehat{x}_{i j-1}, \widehat{x}_{i-1 j}, y_{i j}^{c}, y_{i j}^{s}\right),
$$

where $\widehat{x}_{i j-1}$ and $\widehat{x}_{i-1 j}$ are the two previous neighbor estimates.

Invoking Bayes law,

$$
\begin{aligned}
& p\left(x_{i j} \mid \widehat{x}_{i j-1}, \widehat{x}_{i-1}, y_{i j}^{c}, y_{i j}^{s}\right)= \\
& \quad p\left(x_{i j} \mid \widehat{x}_{i j-1}, \widehat{x}_{i-1 j}\right) \cdot p\left(y_{i j}^{c}, y_{i j}^{s} \mid x_{i j}\right) .
\end{aligned}
$$

In stochastic filtering terminology, expression (9) states that the prediction density $p\left(x_{i j} \mid \widehat{x}_{i j-1}, \widehat{x}_{i-1 j}\right)$ is multiplied by the observation factor $p\left(y_{i j}^{c}, y_{i j}^{s} \mid x_{i j}\right)$, to give the filtering density $p\left(x_{i j} \mid \widehat{x}_{i j-1}, \widehat{x}_{i-1}, y_{i j}^{c}, y_{i j}^{s}\right)$. Accordingly, the proposed scheme proceeds recursively as follows: 
Prediction step Following (2), and taking $x_{i j-1}=\widehat{x}_{i j-1}$ and $x_{i-1 j}=\widehat{x}_{i-1}$

$$
p\left(x_{i j} \mid \widehat{x}_{i j-1}, \widehat{x}_{i-1 j}\right)=\mathcal{N}\left(\frac{1}{2} \widehat{x}_{i j-1}+\frac{1}{2} \widehat{x}_{i-1 j}, \mu^{2}\right),
$$

where $\mathcal{N}\left(a, \mu^{2}\right)$ stands for a Gaussian density of mean $a$ and variance $\mu^{2}$; in the sequel, we shall denote the mean of $(10)$ as $\widehat{\widehat{x}}_{i j} \equiv \frac{1}{2} \widehat{x}_{i j-1}+\frac{1}{2} \widehat{x}_{i-1} j$.

Filtering step As stated in (9), the prediction density is updated by multiplying it by the observation factor. The estimate is obtained by maximizing the resulting filtering density.

\subsection{Nonlinear algorithm}

It was shown in [12] that $p\left(y_{i j}^{c}, y_{i j}^{s} \mid x_{i j}\right)$ (the positive periodic function of $x_{i j}$ given by (5)) is well represented by a train of Gaussian functions

$$
\tilde{p}\left(y_{i j}^{c}, y_{i j}^{s} \mid x_{i j}\right) \propto \sum_{k=-\infty}^{+\infty} \mathcal{N}\left(\eta_{i j}+2 k \pi, \gamma_{i j}^{2}\right),
$$

with common variance $\gamma_{i j}^{2}$ obtained by minimizing the Kullback distance between the normalized central periods of $p\left(y_{i j}^{c}, y_{i j}^{s} \mid x_{i j}\right)$ and of its representation $\tilde{p}\left(y_{i j}^{c}, y_{i j}^{s} \mid x_{i j}\right)$. A numerically obtained lookup table provides the optimal $\gamma_{i j}$ as function of $\lambda_{i j}$ with a minor computational effort [12].

Substituting $p\left(y_{i j}^{c}, y_{i j}^{s} \mid x_{i j}\right)$ by $\tilde{p}\left(y_{i j}^{c}, y_{i j}^{s} \mid x_{i j}\right)$, in (9), leads to a weighted sum of Gaussian functions (still all with a common variance $\nu_{i j}^{2}$ ),

$$
\tilde{p}\left(x_{i j} \mid \widehat{x}_{i j-1}, \widehat{x}_{i-1 j}, y_{i j}^{c}, y_{i j}^{s}\right)=\sum_{k=-\infty}^{\infty} W_{i j}^{k} \mathcal{N}\left(\zeta_{i j}^{k}, \nu_{i j}^{2}\right)
$$

where the weights verify

$$
W_{i j}^{k} \propto \exp \left\{-\frac{\left(\hat{\widehat{x}}_{i j}-\left(\eta_{i j}+2 k \pi\right)\right)^{2}}{2\left(\mu^{2}+\gamma_{i j}^{2}\right)}\right\}
$$

and the means are given by

$$
\zeta_{i j}^{k}=\frac{\widehat{\widehat{x}}_{i j} \gamma_{i j}^{2}+\left(\eta_{i j}+2 k \pi\right) \mu^{2}}{\mu^{2}+\gamma_{i j}^{2}} .
$$

Since the variance is the same for all Gaussians in (12), the one with the highest maximum is simply the one with the largest weight, i.e. the one with mean closest to the prediction density mean $\widehat{\widehat{x}}_{i j}$. Neglecting the small shift induced by lateral terms on the location of the maximum, the estimate is

$$
\widehat{x}_{i j}=\zeta_{i j}^{k^{\star}},
$$

with

$$
\begin{aligned}
k^{*} & =\arg \min _{k}\left\{\left(\hat{\widehat{x}}_{i j}-\left(\eta_{i j}+2 k \pi\right)\right)^{2}\right\} \\
& =\operatorname{round}\left[\frac{\widehat{\hat{x}}_{i j}-\eta_{i j}}{2 \pi}\right] .
\end{aligned}
$$

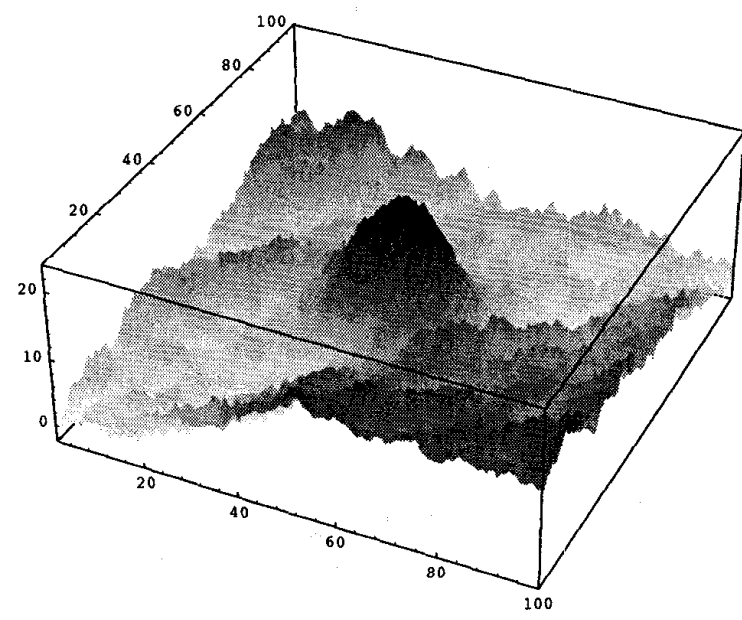

Figure 5: Surface reconstructed by the nonlinear algorithm.

Fig. 5 shows the reconstructed phase image provided by the nonlinear algorithm from the noisy observations of Fig. 3. Notice the ability to follow the true phase surface, even though it contains a deterministic component (the previously mentioned Gaussian-shaped elevation) which is not taken into account in the prior model. This reveals a certain degree of robustness against model mismatch.

\subsection{Linearized Algorithm}

The classical solution to phase tracking is the phase locked loop, which is equivalent to the extended Kalman filter (EKF) applied to the class of models herein considered [11]. In the EKF there is no representation of the observation factor as in (11); instead, the nonlinear observation model is linearized, in each step, around the mean of the prediction density. As a consequence, the observation factor looses its periodic structure, reducing to the Gaussian form

$$
\bar{p}\left(y_{i j}^{c}, y_{i j}^{s} \mid x_{i j}\right) \propto \mathcal{N}\left(\xi_{i j}, \sigma^{2}\right)
$$

where

$$
\xi_{i j}=\widehat{\widehat{x}}_{i j}+y_{i j}^{s} \cos \left(\widehat{\widehat{x}}_{i j}\right)+y_{i j}^{c} \sin \left(\widehat{\widehat{x}}_{i j}\right) .
$$

Instead of becoming multimodal, the filtering density has now a single Gaussian term

$$
\bar{p}\left(x_{i j} \mid \widehat{x}_{i j-1}, \widehat{x}_{i-1 j}, y_{i j}^{c}, y_{i j}^{s}\right)=\mathcal{N}\left(\varphi_{i j}, \psi_{i j}^{2}\right)
$$

with variance $\psi_{i j}^{2}$ and mean given by

$$
\varphi_{i j}=\widehat{\widehat{x}}_{i j}+\frac{\mu^{2}}{\mu^{2}+\sigma^{2}}\left(y_{i j}^{s} \cos \left(\widehat{\widehat{x}}_{i j}\right)+y_{i j}^{c} \sin \left(\widehat{\widehat{x}}_{i j}\right)\right) .
$$

The maximum of (20) is then simply its mean,

$$
\widehat{x}_{i j}=\varphi_{i j} .
$$

Fig. 6 shows the surface produced by this linearized estimator based on the same noisy observations of Fig. 3 
and with the same parameter settings; it is very close to the true surface, except in the central region where it fails to accompany the steep ascent.

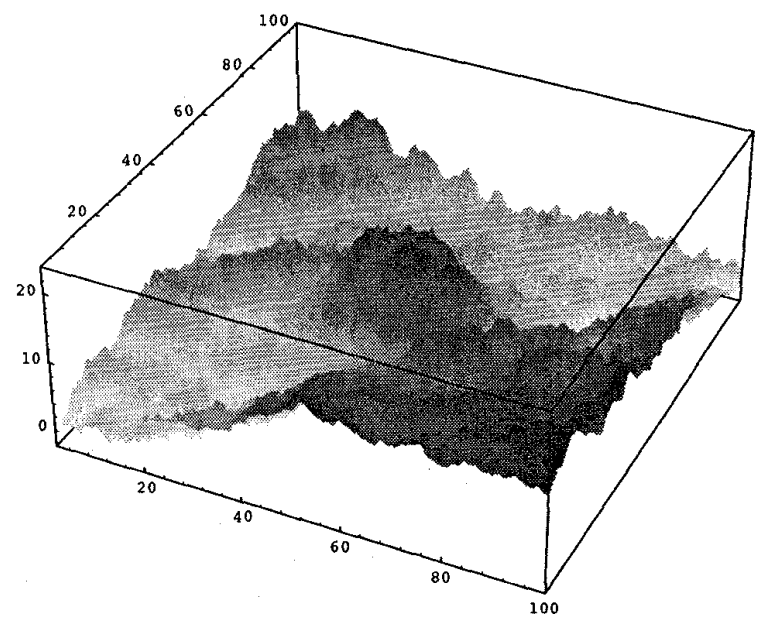

Figure 6: Surface estimate produced by the linearized algorithm.

Fig. 7 , plots the central cross sections of the original surface and of its estimates obtained according to (15) and (22); it is apparent that, besides the serious error in the middle (of about $2 \pi$ ), the linearized algorithm also produces a slightly oversmoothed estimate (when compared with the nonlinear one).

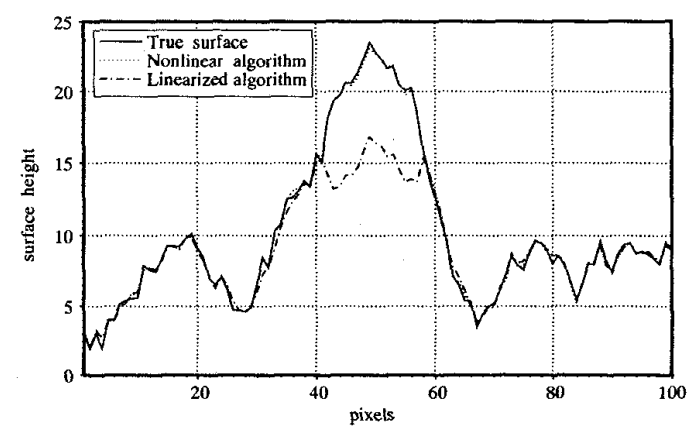

Figure 7: Central cross sections of the original surface and of its estimates, obtained by the two considered algorithms.

\section{FINAL REMARKS}

Whereas the observation model captures the essential features of interferometric imaging, the assumed image prior can be considered simplistic. The reasons that led to its adoption are severalfold: it formalizes (in Bayesian estimation context) surface continuity; it yields feasible algorithms; it can easily be generalized in a variety of directions.

Future research will consider: non-causal GMRF priors and discontinuity detection, where approaches related to the ones proposed in [13] and [14] will be pursued. Cycle slipping, a phenomenon common to all phase estimation problems, may raise additional difficulties which will have to be addressed.

\section{REFERENCES}

[1] E. Rodriguez and J. Martin, "Theory and design of interferometric synthetic aperture radars", IEE Proceedings- $F$, vol. 139, pp. 147-159, 1992.

[2] S. Madsen, H. Zebker, and J. Martin, "Topographic mapping using radar interferometry: Processing techniques", IEEE Trans. on Geosc. and Remote Sens., vol. 31, pp. 246-256, 1993.

[3] S. Pandit, N. Jordache, and G. Joshi, "Data-dependent systems methodology for noise-insensitive phase unwrapping in laser interferometric surface characterization", Jour. of the Optic. Soc. of Amer, vol. 11, pp. 2584-2592, 1994.

[4] J. Gu, Y. Hung, and F. Chen, "Iteration algorithm for computer-aided speckle interferometry", Applied Optics, vol. 33, pp. 5308-5317, 1994.

[5] S. Song, S. Napel, N. Pelc, and G. Glover, "Phase unwrapping of MR phase images using Poisson equation", IEEE Trans. on Image Proc., vol. 4, pp. 667-676, 1995.

[6] N. Ching, D. Rosenfeld, and M. Braun, "Twodimensional phase unwrapping using a minimum spanning tree", IEEE Trans. on Image Proc., vol. 1, pp. 355-365, 1992.

[7] M. Kaveh and M. Soumekh, "Computer-assisted diffraction tomography", in H. Stark, editor, Image Recovery: Theory and Application, pp. 369-413. Academic Press, 1987.

[8] J. Woods, "Two-dimensional discrete Markovian fields", IEEE Trans. on Inform. Theo, vol. 18, pp. 232-240, 1972.

[9] R. Chellappa, "Two-dimensional discrete Gaussian Markov random field models for image processing", in L. Kanal and A. Rosenfeld, editors, Progress in Pattern Recognition. Elsevier Publ., 1985.

[10] J. Besag, "On the statistical analysis of dirty pictures", Jour. of the Roy. Stat. Soc. B, vol. 48, pp. 259-302, 1986.

[11] J. Leitão and J. Moura, "Acquisition in phase demodulation: Application to ranging in radar/sonar systems", IEEE Trans. on Aerosp. and Electr. Syst., vol. 31, 1995.

[12] J. Leitão and J. Moura, "Nonlinear phase estimation based on the Kullback distance", in Proceedings of ICASSP'g4, pp. 521-524, Adelaide, Autralia, 1994.

[13] M. Figueiredo and J. Leitão, "Simulated tearing: an algorithm for discontinuity preserving visual suraface reconstruction", in Proceedings of CVPR'93, pp. 28 33, New York, 1993.

[14] M. Figueiredo and J. Leitão, "Adaptive discontinuity location in image restoration", in Proccedings of ICIP'94, pp. II.665-II.669, Austin, 1994. 